\title{
Sulle equazioni ellittiche del secondo ordine a coefficienti continui.
}

\author{
Maurizlo Chicco (Genova) (*) (**a)
}

Summary. - It is considered a linear second order uniformly elliptic partial differential equation, where coeffeients of second derivatives are supposed uniform7y continuous and the other ones belong to suitable $L_{p}$ classes. I proce some result about existence and uniqueness of the solution of the Dirichlet problem in the space $H^{2}(\Omega) \cap H^{1}{ }_{0}(\Omega)$.

\section{1. - Introduzione.}

Si consideri l'operatore ellittico

$$
L=-\sum_{i, j=1}^{n} a_{i j} \frac{\partial^{2}}{\partial x_{i} \partial x_{j}}+\sum_{i=1}^{n} b_{i} \frac{\partial}{\partial x_{i}}+c
$$

ove i coefficienti $a_{i j}$ sono uniformemente continui nell'aperto $\Omega$ e glì altri coefficienti appartengono ad opportune classi $L_{p}(\Omega)$. Vari autori (vedi ad esempio [1], [8], [10]) hanno studiato maggiorazioni del tipo

$$
\|u\|_{H^{2}(\Omega)} \leq K_{1}\left[\|L u\|_{L_{2}(\Omega)}+\|u\|_{L_{2}(\Omega)}\right]
$$

valida per ogni funzione $u$ nulla sulla frontiera di $\Omega$ e dotata di derivate seconde (generalizzate) di quadrato sommabile in $\Omega$. La costante $K_{1}$ dipende da $\Omega$ e dai coefficienti di (1). Non mi risulta tuttavia ohe la disuguaglianza (2) sia finora stata sfruttata per risolvere il seguente problema di DrRoHLEx: assegnata comunque nna funzione $f \in L_{2}(\Omega)$, stabilire l'esistenza di una funzione $u$, dotata di derivate seconde (generalizzate) di quadrato sommabile in $\Omega$ e tale che

$$
\begin{cases}L u=f & \text { q.o. in } \Omega, \\ u=0 & \text { su } \partial \Omega .\end{cases}
$$

(*) Lavoro eseguito nell' ambito del centro di ricerca di matematica e fisica teorica del Consiglio Nazionale delle Ricerche presso l'Universita di Genova.

(**) Entrata in Redazione il 13 settembre 1970. 
124 Maurizio Chicco: Sulle equazioni ellittiche del secondo ordine, ecc.

Nel presente lavoro dimostro che, sotto opportune ipotesi sul coefficiente $c$ di $L$, il problema (3) ammette una ed una sola soluzione.

\section{2. - Notazioni ed ipotesi.}

Salvo avviso contrario nel seguito si faranno sempre le seguenti ipotesi. Sia $\Omega$ un insieme aperto limitato di $R^{n}$, con $n \geq 2$. Supponiamo che la frontiera di $\Omega$ sia rappresentabile localmente come grafico di una funzione dotata di derivate seconde continue. Siano $H^{1}(\Omega)$ ed $H_{0}^{1}(\Omega)$ gli spazi ottenuti completando rispettivamente $C^{1}(\bar{\Omega})$ e $C_{0}^{1}(\Omega)$ secondo la norma

$$
\|u\|_{H^{1}(\Omega)}=\|u\|_{L_{2}(\Omega)}+\sum_{i=1}^{n}\left\|u_{x_{i}}\right\| L_{L_{2}(\Omega)}
$$

In $H_{0}^{1}(\Omega)$ una norma equivalente è la seguente:

$$
\left\|u_{x}\right\|_{L_{i}(\Omega)}=\left\{\sum_{i=1}^{n}\left\|u_{x_{i}}\right\|_{L_{2}(\Omega)}^{2}\right\}^{\ell}
$$

Sia $H^{2}(\Omega)$ lo spazio ottenuto completando $C^{2}(\bar{\Omega})$ secondo la norma

$$
\|u\|_{H^{2}(\Omega)}=\|u\|_{L_{2}(\Omega)}+\sum_{i, j=1}^{n}\left\|u_{x_{i}^{*} j}\right\|_{L_{\varepsilon}(\Omega)}
$$

Poniamo

$$
\boldsymbol{u}_{x x}=\left\{\sum_{i, j=1}^{n} \boldsymbol{u}_{x_{i} x_{j}}^{2}\right\}^{\frac{1}{2}}
$$

e osserviamo che in $H^{2}(\Omega) \cap H_{0}^{1}(\Omega)$ le quantità $\|u\|_{H^{2}(\Omega)}$ e $\left\|u_{x x}\right\|_{L_{2}(\Omega)}$ sono norme equivalenti (redi ad esempio [14] pag. 288). Siano poi $a_{i j} \in C^{0}(\bar{\Omega})$, $\sum_{i, j=1}^{n} a_{i j} t_{i} t_{j} \geq v|t|^{2}, \nu$ costante positiva, $a_{i j}=a_{j i}(i, j=1,2, \ldots, n), b_{i} \in L_{p}(\Omega)$ $(i=1,2, \ldots, n)$ con $p>2$ se $n=2, p=n$ se $n \geq 3 ; c \in L_{q}(\Omega)$ con $q=2$ se $2 \leq n \leq 3, q>2$ se $n=4, q=n / 2$ se $n \geq 5$. Sia infine $L$ l'operatore definito nella (1).

\section{3. - Risultati.}

$\underset{t}{\text { Lemma } 1 . ~-~}$ Siano $a_{i j}^{0}(i, j=1,2, \ldots, n)$ costanti tali che $\sum_{i, j=1}^{n} a_{i j}^{0} t_{i} t_{j} \geq$ $\geq v|t|^{2}$ e sia

$$
L_{0}=-\sum_{i, j=1}^{n} a_{i j}^{0} \frac{\partial^{2}}{\partial x_{i} \partial x_{j}}+\sum_{i=1}^{n} b_{i} \frac{\partial}{\partial x_{i}}+c
$$


Allora esistono due costanti positive $K_{2}$ e $\lambda_{0}$, dipendenti da $\vee, \Omega$ e dai coefficienti $b_{i}(i=1,2, \ldots, n), c$ di $L_{0}$ tali che risulti

$$
\left\|\boldsymbol{u}_{x x}\right\|_{L_{2}(\Omega)} \leq K_{2}\left[\left\|\left(L_{0}+\lambda I\right) u\right\|_{L_{2}(\Omega)}+\|u\|_{L_{2}(\Omega)}\right]
$$

per ogni $u \in H^{2}(\Omega) \cap H_{0}^{1}(\Omega)$ ed uniformemente per ogni $\lambda \geq \lambda_{0} »$.

Dimostrazione. - Per [11] pag. 169.183 sussiste una disnguaglianza del tipo

$$
\left\|u_{x x}\right\|_{L_{2}(\Omega)} \leq K_{3}\left[\left\|L_{0} u\right\|_{L_{2}(\Omega)}+\|u\|_{L_{2}(\Omega)}\right]
$$

valida per ogni $u \in H^{2}(\Omega) \cap H_{0}^{1}(\Omega)$ e con $K_{3}$ dipendente da $\Omega$ e dai coefficienti di $L_{0}$. Risulta poi, per le stesse funzioni $u$ :

(5)

$$
\left\|\left(L_{0}+\lambda I\right) u\right\|_{L_{2}(\Omega)}^{2}=\left\|L_{0} u\right\|_{L_{-}(\Omega}^{2}+\lambda^{2}\|u\|_{L_{2}(\Omega)}^{2}+2 \lambda \int_{\Omega}\left(L_{0} u\right) u d x=
$$

$=\left\|L_{0} u\right\|_{L_{2}(\Omega)}^{2}+\lambda^{2}\|u\|_{L_{2}(\Omega)}^{2}+2 \lambda \int_{\Omega}\left\{-\sum_{i_{, j}=1}^{n} a_{i j}^{0} u_{x_{i} x_{j}} u+\sum_{i=1}^{n} b_{i} u_{x_{i}} u+c u^{2}\right\} d x$.

Per le ipotesi fatte e per le formule di GraEn si ha

$$
-\int_{\Omega} \sum_{i, j=1}^{n} a_{i j}^{0} u_{x_{i}, j} u d x=\int_{\Omega} \sum_{i, j=1}^{n} a_{i j}^{0} u_{x_{i}} u_{x_{j}} d x \geq \nu\left\|u_{x}\right\|_{L_{y}(\Omega)}^{2},
$$

e inoltre note disuguaglianze (vedi ad esempio [ic] teorema 5.1) implicano che per ogni $\varepsilon>0$ risulti

$$
\begin{gathered}
\sum_{i=1}^{n}\left|\int_{\Omega} b_{i} u_{x_{i}} u d x\right| \leq \varepsilon\left\|u_{x}\right\|_{L_{2}(\Omega)}^{2}+K_{4}\|u\|_{L_{i}(\Omega)}^{2}, \\
\left|\int_{\Omega} c u^{2} d x\right| \leq \varepsilon\left\|u_{x}\right\|_{L_{2}(\Omega)}^{2}+K_{5}\|u\|_{L_{2}(\Omega)}^{2},
\end{gathered}
$$

ove le costanti $K_{4}, K_{5}$ dipendono da $\varepsilon$ e dai coefficienti $b_{i}(i=1,2, \ldots, n)$, c. Allora scelto $\varepsilon=\nu / 4$ e posto $\lambda_{0}=2\left(K_{4}+K_{5}\right)$ dalle $(5), \ldots$, (8) segue che se $\lambda \geq \lambda_{0}$ risulta

$$
\left\|L_{0} u\right\|_{L_{2}(\Omega)} \leq\left\|\left(L_{0}+\lambda I\right) u\right\|_{L_{2}(\Omega)}, \quad \forall u \in H^{2}(\Omega) \cap H_{0}^{1}(\Omega) .
$$

Dalle (4) $\theta(9)$ è immediata la tesi, c.v.d. 
Lemma 2. - "Esistono due costanti positive $\lambda_{0} e K_{6}$ dipendenti dai coefficienti di $L$, da n e da $\Omega$, tali che risulti

$$
\left\|u_{x x}\right\|_{L_{2}(\Omega)} \leq K_{6}\left[\|(L+\lambda I) u\|_{L_{2}(\Omega)}+\|u\|_{L_{2}(\Omega)}\right]
$$

per ogni $u \in H^{2}(\Omega) \cap H_{0}^{1}(\Omega)$ e uniformemente per ogni $\lambda \geq \lambda_{0}$ »

Drmostrazione. - Basta ripetere parola per parola quella di [11] pag. 190.193 , osservando che quando si trovano disuguaglianze di tipo (4) in sottoinsiemi di $\Omega$ di diametru piccolo per operatori a coefficienti costanti che approssimano $L$, tali disuguaglianze valgono uniformemente per $\lambda \geq \lambda_{0}$ come si è visto nel lemma precedente.

Per convenienza del lettore la dimostrazione dettagliata è riportata in appendice.

Teorema 1. - «Esistono due costanti positive $K_{\bar{\tau}}$ e $\lambda^{*}$ dipendenti dai coefficienti di $L$, da $n$ e da $\Omega$, tali che risulti

$$
\left\|u_{x x}\right\|_{L_{2}(\Omega)} \leq K_{7}\|(L+\lambda I) \boldsymbol{u}\|_{L_{2}(\Omega)}
$$

per ogni $u \in H^{2}(\Omega) \cap H_{0}^{1}(\Omega)$ e uniformemente per ogni $\lambda \geq \lambda^{*}$ ».

Dimostrazione. - Siano $a_{i j}^{0}$ ed $L_{0}$ definiti come nel lemma 1 ; poniamo

$$
M=n \max _{\bar{\Omega}} \sum_{i, j=1}^{n}\left|\alpha_{i j}-a_{i j}^{0}\right|
$$

L'operatore $L_{0}$ può scriversi anche in forma variazionale (vedi [12]); per la teoria di tali equazioni svolta da G. Stampacchia ([13] teorema 3.2) esi. stono costanti positive $c_{0}, c_{1}$ tali che risulti

$$
\int_{\Omega}\left(L_{0} u\right) u d x \geq c_{1}\left\|u_{x}\right\|_{L_{3}(\Omega)}^{2}-c_{0}\|u\|_{L_{2}(\Omega)}^{2}
$$

per ogni $u \in H^{2}(\Omega) \cap H_{0}^{1}(\Omega)$. Ne segue

$$
\int_{\Omega}\left(L_{0} u+\lambda \boldsymbol{u} ; \boldsymbol{u} d x \geq c_{1}\left\|u_{x}\right\|_{L_{2}(\Omega)}^{2}+\left(\lambda-c_{0}\right)\|u\|_{L_{0}(\Omega)}^{2}\right.
$$

per ogni $u \in H^{2}(\Omega) \cap H_{0}^{1}(\Omega)$ e per ogni $\lambda>c_{0}$, da cui subito

$$
\|u\|_{L_{2}(\Omega)} \leq\left(\lambda-c_{0}\right)^{-1}\left\|\left(L_{0}+\lambda I\right) u\right\|_{z_{2}(\Omega)} .
$$


Dalla (11) si ottiene

$$
\left\|L_{0} u-L u\right\|_{L_{2}(\Omega)} \leq M\left\|\boldsymbol{u}_{x x}\right\|_{L_{2}(\Omega)}
$$

per ogni $u \in H^{2}(\Omega) \cap H_{0}^{1}(\Omega)$. Utilizziamo ora le (10), (14), (15) osservando che le costanti $K_{6}$ e $c_{0}$ non dipendono da $\lambda$. Per ogni $u \in H^{2}(\Omega) \cap H_{0}^{1}(\Omega)$ e per $\operatorname{ogni} \lambda>\max \left(c_{0}, \lambda_{0}\right)$ si ha:

$$
\begin{gathered}
\left\|u_{x x}\right\|_{L_{2}(\Omega)} \leq K_{6}\left[\|(L+\lambda I) \boldsymbol{u}\|_{L_{2}(\Omega)}+\|u\|_{L_{2}(\Omega)}\right] \leq \\
\leq K_{6}\left[\|(L+\lambda I) u\| L_{L_{2}(\Omega)}+\left(\lambda-c_{0}\right)^{-1}\left\|\left(L_{0}+\lambda I\right) u\right\|_{L_{2}(\Omega)}\right] \leq \\
\leq K_{6}\left[\|(L+\lambda I) u\|_{L_{2}(\Omega)}+\left(\lambda-c_{0}\right)^{-1}\|(L+\lambda I) u\| L_{L_{i}(\Omega)}+M\left(\lambda-c_{0}\right)^{-1}\left\|u_{x x}\right\|_{L_{2}(\Omega)}\right)
\end{gathered}
$$

A questo punto sia $\lambda^{*}=\max \left(\lambda_{0}, 2 M K_{6}+c_{0}\right)$. Allora dalla (16) segue

$$
\left\|u_{x_{x}}\right\|_{L_{L_{2}}(\Omega)} \leq 2 K_{6}\left[1+\left(\lambda-c_{0}\right)^{-1}\right]\|(L+\lambda I) u\|_{L_{2}(\Omega)}
$$

valida per ogni $u \in H^{2}(\Omega) \cap H_{0}^{1}(\Omega)$ ed uniformemente per ogni $\lambda \geq \lambda^{*}$, c.v.d.

Corollario 1. - "Sia $\lambda \geq \lambda^{*}, c \geq 0$ quasi ovunque in $\Omega, f \in L_{2}(\Omega)$, $f \geq 0$ quasi ovunque in $\Omega, u \in H^{2}(\Omega) \cap H_{0}^{1}(\Omega),(L+\lambda I) u=f$ in $\Omega$. Allora risulta $u \geq 0$ quasi ovunque in $\Omega$.

Dimostrazione: si trova nell' appendice.

Teorema 2. - «ia ess inf $c>0$. Allora il problema di Dirichlet

$$
\left\{\begin{array}{l}
L u=f \quad \text { q.o. in } \Omega, \\
u \in H^{2}(\Omega) \cap H_{0}^{\mathrm{l}}(\Omega)
\end{array}\right.
$$

ammette una ed una sola soluzione comunque si assegni $f$ in $L_{2}(\Omega)$. Se per almeno un valore di $i(1 \leq i \leq n)$ è $b_{i} \in L_{\infty}(\Omega)$, il risultato vale anche se ess inf $c=0 »$.

Dimostrazione. - Sia $\lambda \geq \lambda^{*}$, ove $\lambda^{*}$ è definito nel teorema 1. Allora esiste l'operatore inverso di $L+\lambda I$, e sia $G_{\lambda}$, che porta $L_{2}(\Omega)$ in $H^{2}(\Omega) \cap H_{0}^{1}(\Omega)$. Poichè $G_{\lambda}$ è un operatore compatto, il suo spettro è discreto e numerabile; indicando con $\left\{\lambda_{j}\right\}_{j \in N}$ la successione degli autoralori di $L$ e con $\left\{\mu_{j}\right\}_{j \in N}$ quella degli autovalori di $G_{\lambda}$, risulta

$$
\mu_{j}=\frac{1}{\lambda_{j}+\lambda}, \quad j \in N
$$


per ogni $\lambda \geq \lambda^{*}$. Inoltre, per il corollario precedente, all'operatore $G_{\lambda}$ si può applicare il teorema $6.1 \mathrm{di}[9]$ in quanto $G_{\lambda}$ lascia invariante il cono delle funzioni positive di $L_{2} \Omega$ ). Usando un procedimento gia utilizzato in [3], [4] si trova che esiste un autovalore $\mu_{1}$ di $G_{\lambda}$ reale e di modulo massimo tra tutti gli autovalori di $G_{\lambda}$ :

$$
{ }^{\mid} \mu_{j} \mid \leq \mu_{1} \quad \forall \mu_{j} \text { autovalore di } G_{\lambda} .
$$

Dalla (20) segue che esiste in corrispondenza di $\mu_{1}$ l'autovalore (reale) di $L \lambda_{1}=\frac{1-\lambda \mu_{1}}{\mu_{1}}$ tale che

$$
\text { Re } \lambda_{j} \geq \lambda_{1} \quad \forall \lambda_{j} \text { autovalore di } L \text {. }
$$

$\grave{\mathrm{E}}$ chiaro quindi che la tesi sarà provata non appena si dimostri che $\lambda_{1}>0$. A questo scopo consideriamo una successione $\alpha_{i j}^{(m)} \in C^{\infty}(\bar{\Omega})(m=1,2, \ldots)$ tale che

$$
\lim _{m \rightarrow+\infty} \max _{\bar{\Omega}} \sum_{i, j=1}^{n}\left|a_{i j}-a_{i j}^{(m)}\right|=0
$$

e sia

$$
L^{(m)}=-\sum_{i, j=1}^{n} a_{i j}^{(m)} \frac{\partial^{2}}{\partial x_{i} \partial x_{j}}+\sum_{i=1}^{n} b_{i} \frac{\partial}{\partial x_{i}}+c
$$

Siano poi $G_{\lambda}^{(m)}$ gli operatori inversi di $L^{(m)}+\lambda I$, certamente esistenti per $\lambda \geq \lambda^{*}$. Dalla (23) segue

$$
\lim _{m \rightarrow+\infty}\left\|\left(L-L^{(m)}\right) u\right\|_{L_{2}(\Omega)}=0
$$

ove il limite è uniforme per $u \in H^{2}(\Omega) \cap H_{0}^{1}(\Omega),\left\|u_{x x}\right\| r_{2}(\Omega) \leq 1$.

Ciò implica che la successione di operatori $\left\{G_{\lambda}^{(m)}\right\}_{m \in N}$ converge a $G_{\lambda}$ nella metrica uniforme di $\zeta\left[L_{2}(\Omega) ; H^{2}(\Omega) \cap H_{0}^{1}(\Omega)\right]$.

Per il lemma a pag. 1091 di $[6]$ risulta allora

$$
\lim _{m \rightarrow+\infty} \mu_{j}^{(m)}=\mu_{j}
$$

uniformemente per $j \in N$, ove $\left\{\mu_{j}^{(m)}\right\}_{i \in N}$ è la successione degli autovalori di $G_{\lambda}^{(m)}$. In particolare è

da cui subito

$$
\lim _{m \rightarrow+\infty} \mu_{1}^{(m)}=\mu_{1}
$$

$$
\lim _{m \rightarrow+\infty} \lambda_{1}^{(m)}=\lambda_{1}
$$


ove $\lambda_{1}^{(m)}$ è l'autovalore di minima parte reale di $L^{(m)}$, cioè

$$
\lambda_{1}^{(m)}=\frac{1-\lambda \mu_{1}^{(m)}}{\mu_{1}^{(m)}}
$$

Osserviamo a questo punto che l'operatore $L^{(m)}$ si può scrivere anche in forma rariazionale e si possono quindi applicare ad esso i risultati di [13] $\theta[2]$.

In particolare essendosi supposto $c \geq k$ q.o. in $\Omega$ con $k$ costante positiva, da [13] teorema 3.8 segue che $\lambda_{1}^{(m)} \geq k$ per ogni $m=1,2, \ldots$. Dalla (27) si ottiene quindi

$$
\lambda_{1} \geq k>0
$$

Pertanto 0 non è un autovalore di $L$ e il problema (19) ammette una ed una sola soluzione.

Resta da provare che se $b_{i} \in L_{\propto}(\Omega)$ per almeno un valore di $i$, il risultato è vero anche se ess inf $c=0$. A questo scopo basta utilizzare un artificio di PiCARD come in [5] pag. 322. Riporto la dimostrazione per convenienza del lettore.

Sia $u \in H^{2}(\Omega) \cap H_{0}^{1}(\Omega), L u=0$ q.o, in $\Omega$, ess inf $c=0, b_{1} \in L_{\infty}(\Omega)$; facciamo vedere che $u=0$ q.o. in $\Omega$.

Poniamo $u=z v$ con $z=C-e^{\beta x_{1}}$; le quantità $\mu$ e $C$ sono costanti da fissare più tardi.

Si ha:

$$
\begin{gathered}
L u=-\sum_{i, j=1}^{n} a_{i j} z_{x_{i} x_{j}} v-\sum_{i, j=1}^{n} a_{i j} v_{x_{i} x_{j}} z-2 \sum_{i, j=1}^{n} a_{i j} z_{x_{i}} v_{x_{j}}+ \\
+\sum_{i=1}^{n} b_{i} z_{x_{i}} v+\sum_{i=1}^{n} b_{i} v_{x_{i}} z+c z v=0
\end{gathered}
$$

e per le posizioni fatte

$$
\begin{array}{cl}
z\left[-\sum_{i, j=1}^{n} a_{i j} v_{x_{i} x_{j}}+\left(\frac{2 \mu \mu^{\mu x_{1}}}{z} \sum_{i=1}^{n} a_{i 1}+\sum_{i=1}^{n} b_{i}\right) v_{x_{i}}+\right. & \\
\left.+\left(\frac{a_{11} \mu^{2} e^{\mu x_{1}}-b_{1} \mu e^{\mu x_{1}}}{z}+c\right) v\right]=0 & \text { q.o. in } \Omega .
\end{array}
$$

Si scelgono ora le costanti $C$ e $\mu$ in modo che $z>1$ in $\Omega \theta$ $\underset{\Omega}{\operatorname{ess}} \inf \left(\frac{a_{11} \mu^{2} e^{\mu x_{1}}-b_{1} \mu e^{\mu x_{1}}}{z}+c\right)>0$.

Allora l'equazione (29) diventa del tipo 


$$
\widehat{L} v=0
$$

q.o. in $\Omega$

ove i coefficienti soddisfano alle ipotesi necessarie per applicare la prima parte del teorema. Ne segue $v=0$ q.o. in $\Omega$ e quindi $u=0$ q.o. in $\Omega$. Percio di nuovo 0 non è un autovalore di $L$ e il problema (19) ammette una ed una sola soluzione, c.v.d.

Corollario 2. - "Siano soddisfalte le ipotesi del teorema precedente. Allora esiste una costante $K_{8}$ indipendente da u tale che

$$
\left\|u_{x x}\right\| L_{x_{2}(\Omega)} \leq K_{8}\|L u\|{\underline{x_{2}(\Omega)}}_{2}
$$

per ogni $u \in H^{2}(\Omega) \cap H_{0}^{1}(\Omega)$. Se inoltre $\dot{e}$ Lu $\geq 0$ q.o. in $\Omega$, ne segue $u \geq 0$ q.o. in $\Omega$ ».

Dimostrazrone. - La disuguaglianza $(*)$ segue subito dal fatto che, per il teorema precedente, lo zero non è un antovalore di $L$ e quindi esiste l'operatore $L^{-1}$ (inverso di $L$ ) limitato da $L_{2} \Omega^{\prime}$ ) ad $H^{2}(\Omega) \cap H_{0}^{\mathrm{l}}(\Omega)$.

L'ultima affermazione si deduce nel modo seguente: detti $L^{(m)}$ gli operatori definiti nella dimostrazione del teorema precedente, sinno $u^{(m)}$ le soluzioni dei problemi di DIRIOHLET

$$
\left\{\begin{array}{l}
L^{(m)} u^{(m)}=L u \quad \text { q.o. in } \Omega \\
u^{(m)} \in H^{2}(\Omega) \cap H_{0}^{1}(\Omega) .
\end{array}\right.
$$

Per noti teoremi (vedi ad esempio [2]) se è $L u \geq 0$ quasi ovunque in $\Omega$ risulta $u^{(m)} \geq 0$ quasi orunque in $\Omega$.

Per le (25), (30) si ottiene facilmente

$$
\lim _{m \rightarrow \infty}\left\|u^{(m)}-u\right\|_{L_{2}(\Omega)}=0
$$

da cui la tesi, c.v.d.

\section{4. - Appendice.}

Dimostrazione dec lemma 2. - Per la supposta uniforme continuità in $\Omega$ dei coefficienti $\alpha_{i j}$, per ogni $\varepsilon>0$ eे possibile determinare un numero $R>0$ e dei punti $x_{1}, x_{2}, \ldots, x_{q} \in \Omega$ tali che, posto

risulti

$$
S(y, r)=\left(x: \mid x-y_{1}<r\right)
$$

$$
\Omega \subset \bigcup_{l=1}^{q} S\left(x_{l}, R\right)
$$




$$
\max _{1 \leq l \leq q} \max _{x \in S\left(x_{t}, 2 R\right)} \sum_{i, j=1}^{n}\left|a_{i j}(x)-a_{i j}\left(x_{l}\right)\right|<\varepsilon .
$$

Siano poi $\varphi_{1}, \varphi_{2}, \ldots, \varphi_{q}$ funzioni tali che: $\varphi_{l} \in C_{0}^{\infty}\left(S\left(x_{l}, 2 R\right)\right), \quad 0 \leq \varphi_{l} \leq 1$, $\varphi_{l} \equiv 1$ in $S\left(x_{l}, R\right)($ per $l=1,2, \ldots, q)$.

È chiaro allora che $\varphi_{l} u \in H^{2}\left(\Omega \cap S\left(x_{l}, 2 R\right)\right) \cap H_{0}^{1}\left(\Omega \cap S\left(x_{l}, 2 R\right)\right)$ per ogni $u \in H^{2}(\Omega) \cap H_{0}^{1}(\Omega)$.

Posto

$$
L_{0}^{(l)}=-\underbrace{n}_{i, j=1} a_{i j}\left(x_{l}\right) \frac{\partial^{2}}{\partial x_{i} \partial x_{j}}+\sum_{i=1}^{n} b_{i} \frac{\hat{c}}{\partial x_{i}}+c
$$

per la (9) risulta

$$
\left\|L_{0}^{(l)}\left(\varsigma_{l} u\right)\right\|_{L_{g}(\Omega)} \leq\left\|\left(L_{0}^{(l)}+\lambda I\right)\left(\varphi_{l} \boldsymbol{u}\right)\right\|_{L_{g}(\Omega)}
$$

per ogni $u \in H^{2}(\Omega) \cap H_{0}^{1}(\Omega)$ e per ogni $\lambda \geq \lambda_{0}$ (ricordiamo che $\lambda_{0}$ dipende solo dai coefficienti $b_{i}, c$ ). Per noti teoremi (vedi ad esempio [11] lemma 8.1) si ha

$$
\left\|\varphi_{i} u\right\|_{H^{2}(\Omega)} \leq K_{9}\left\{\left\|L_{0}^{(l)}\left(\varphi_{l} u\right)\right\|_{L_{2}(\eta)}+\left\|\varphi_{i} \boldsymbol{u}\right\|_{L_{2}(\Omega)}\right\} \quad(l=1,2, \ldots, q) .
$$

La costante $K_{9}$ dipende solo da $b_{i}, c, \gamma, n$ e dalle curvature di $\partial \Omega$ e quindi non dipende da $l, q, \varepsilon, R$.

Per le (33), (34) si ottiene:

$$
\left\|\varphi_{i} u\right\|_{H^{2}(\Omega)} \leq K_{9}\left(\left\|\left(L_{0}^{(l)}+\lambda I\right)\left(\varphi_{1} u\right)\right\|_{L_{2}(\Omega)}+\left\|\varphi_{l} u\right\|_{L_{2}(\Omega)}\right)
$$

per ogni $\lambda \geq \lambda_{0}$. Dalle $(32),(35)$ segue

$$
\left\|\varphi_{l} u\right\|_{H^{2}(\Omega)} \leq \boldsymbol{K}_{9}\left\{\left\|(L+\lambda I)\left(\varphi_{l} u\right)\right\|_{L_{2}(\Omega)}+\left\|\varphi_{l} u\right\|\left\|_{L_{2}(\Omega)}+n \varepsilon\right\| \varphi_{l} u \|_{H^{2}(\Omega)}\right\} .
$$

Fissiamo ora $\varepsilon=\left(2 n K_{9}\right)^{-1}$; in tal modo restano determinati anche $R$ e q. La $(36)$ fornisce

$$
\left\|\varphi_{l} u\right\|_{H^{2}(\Omega)} \leq 2 K_{9}\left\{\left\|(L+\lambda I)\left(\varphi_{l} u\right)\right\|_{L_{2}(\Omega)}+\left\|\varphi_{l} u\right\| L_{L_{2}(\Omega)}\right\} .
$$

A questo punto un facile calcolo mostra che

$$
\left\|(L+\lambda I)\left(\varphi_{1} u\right)\right\|_{L_{2}(\Omega)} \leq\|(L+\lambda I) u\|_{L_{2}(\Omega)}+K(l)\left[\left\|u_{x}\right\|_{L_{2}(\Omega)}+\|u\|_{L_{2}(\Omega)}\right] .
$$

La costante $K(l)$ dipende da $\varphi_{I}$ e dai coefficienti di $L$; poniamo

$$
K_{10}=\max _{1 \leq l \leq q} K(l)
$$


132 Maurizio Chicco: Sulle equazioni ellitiche del secondo ordine, ecc.

Osserviamo inoltre che, per la scelta delle funzioni $\varphi_{l}$, è:

$$
\begin{gathered}
\left\|\varphi_{l} u\right\|_{L_{2}(\Omega)} \leq\|u\|_{L_{2}(\Omega)} \quad(l=1,2, \ldots, q), \\
\|u\|_{H_{2}(\Omega)} \leq \sum_{l=1}^{q}\left\|\varphi_{l} u\right\|_{H^{2}(\Omega)}+K_{11}\left[\|u\|_{L_{2}(\Omega)}+\left\|u_{x}\right\| L_{L_{2}(\Omega)}\right]
\end{gathered}
$$

ove la costante $K_{11}$ dipende dal massimo modulo delle derivate (prime e seconde) delle funzioni $\varphi_{l}$.

Dalle (37), ..., (41) segue

$$
\|\boldsymbol{u}\|_{H^{2}(\Omega)} \leq 2 q K_{9}\left(K_{10}+K_{11}+1\right)\left[\left\|u_{x}\right\|_{L_{2}(\Omega)}+\|u\|_{L_{2}(\Omega)}+\|(L+\lambda I) u\|_{L_{9}(\Omega)}\right] .
$$

Infine utilizzando la nota disuguaglianza

$$
\left\|\boldsymbol{u}_{x}\right\|_{L_{2}(\Omega)} \leq \eta\|\boldsymbol{u}\|_{H^{2}(\Omega)}+C(\eta)\|\boldsymbol{u}\|_{L_{2}(\Omega)} \quad \forall \eta>0
$$

e la (42) si ottiene la (10), valida per ogni $\lambda \geq \lambda_{0}$ e por ogni $u \in H^{2}(\Omega) \cap$ $\cap H_{0}^{1}(\Omega)$, c.v.d.

Dimostrazione del corollario 1. - Si considerino gli operatori $L^{(m)}$ definiti nelle $(23),(24)$. Sia $u^{(m)}$ la soluzione, per $m=1,2, \ldots$, e per $\lambda \geq \lambda^{*}$, del problema di DiRiohlet

$$
\left\{\begin{array}{l}
\left(L^{(m)}+\lambda I\right) u^{(m)}=f \text { q.o. in } \Omega, \\
u^{(m)} \in H^{2}(\Omega) \cap H_{0}^{1}(\Omega) .
\end{array}\right.
$$

Per le ipotesi fatte e per i risultati di [2], [13] tale soluzione $u^{(m)}$ esiste ed è unica, ed inoltre risulta

$$
u^{(m)} \geq 0 \quad \text { in } \Omega, \text { per } \boldsymbol{m}=1,2, \ldots
$$

Applicando il teorema 1 all'operatore $L^{(m)}$ si trova

$$
\left\|u_{x x}^{(m)}\right\| L_{L_{2}(\Omega)} \leq K_{12}\|f\|_{L_{2}(\Omega)} \quad(m=1,2, \ldots)
$$

ed è facile verificare (rivedendo le dimostrazioni precedenti) che la costante $K_{12}$ può essere scelta indipendente da $m$.

Dalla (45) segue che una successione estratta dalla $u^{(m)}$ converge debolmente ad una funzione $v$ che per le (43) è soluzione della equazione $(L+\lambda I) v=f$. Per il teorema 1 è $u=v$ e per la (44) si ottiene $u \geq 0$ q.o. in $\Omega$, c.v.d. 


\section{BIBLIOGRAFIA}

[1] R. Caccoppon, Limitazioni integrali per le soluzioni di una equazione ellitica alle derivate parziali, Giorn. Mat. Battaglini (4), vol. 4 (1951), pag. 186.212.

[2] M. CHIcco, Principio di massimo forte per sottosoluzioni di equazioni ellitiche di tipo variazionale, Bollettino dell' Unione Matematica Italiana (3), vol. 22 (1967), pag. 368-372.

[B] - - Equazioni ellittiche del secondo ordine di tipo Cordes con termini di ordine inferiore. Annali di Matematica Pura e Applicata (4), vol. 85 (1970), pag. 347.356.

[4] - -, Principio di massimo generalizzato e valutazione del primo autovalore per equa. zioni ellitiche del secondo ordine in forma variazionale, Annali di Matematica Pura ed Applicata (4), rol. 87 (1970), pag. 1-10.

[5] R. Courant, D. Hilbert, Methods of mathematical physics, vol. 2, Interscience, New York (1962).

[6] N. Dunford, J.T. SChwartz, Linear operators, part. 2, Interscience, New York (1963).

[7] E. Gagliardo, Proprietò di alcune classi di funzioni in più variabili, Ricerche di Matematica, vol. 7, (1953), pag. 102-137.

[8] A. I. Koseuev, A priori estimate in $L_{\beta}$ and generalized solutions of elliptic equations and systems, American Mathematical Society Translations (2), vol. 20 (1962), pag. $10 \overline{\text {. }} 171$.

[9] M.G. Krexn, N.A. RUTMax, Linear operators leaving invariant cone in a Banach space, Amerioan Mathematical Society Traslations (1), vol. 10 (1962), pag. 199.325.

[10] O. A. Ladyzhenskata, On the closure of an elliptic operator, Doklady Akad. Nank USSR, vol. 79 (195̃), pag. 723.725 .

[11] - -, N. N. URAL'tseva, Linear and Quasilinear Elliptic Equations, Academic Press, New York (1968).

[12] C. Miranda, Sulle equazioni ellittiche del secondo ordine di tipo non variazionale a coefficienti discontinui, Annali di Matematica Pura ed Applicata (4), vol. 63 (1963), pag. 353.386.

[13] G. Stampacchia, Le problème de Dirichlet pour les équations elliptiques du second ordre $\dot{a}$ coefficients discontinus, Annales Institat Fourier (Grenoble), vol. 15 (1965), pag. 189.258 .

114] G. TaLentr, Sopra una classe di equazioui ellittiche a coefficienti misurabili, Annali di Matematica Pura ed Applicata (4), vol 69 (1965), pag. 285.304. 